\title{
Using Health Information Exchange to Improve Use of Prescription Monitoring Data
}

\author{
Dan Bolton*, Jennifer Sabel, Chris Baumgartner, Travis Kushner and \\ Bryant Thomas Karras
}

Washington State Department of Health, Lacey, WA, USA

\section{Objective}

Demonstrate that use of the Washington State health information exchange (HIE) to facilitate access to prescription monitoring program (PMP) data enhances the effectiveness of a PMP. The increased accessibility will lead to improved patient care by giving providers more complete and recent data on patients' controlled substance prescriptions.

\section{Introduction}

Washington State experienced a five-fold increase in deaths from unintentional drug overdoses between 1998 and 2014. The PMP collects data on controlled substances prescribed to patients and makes the data available to healthcare providers, giving providers another tool for patient care and safety. Optimal impact for the program depends on providers regularly accessing the information to review patients' dispensing history. We have found through provider surveys and work with stakeholders that the best way to increase use is to make data seamlessly accessible through electronic health record systems (EHRs). This approach does not require a separate login to the PMP portal. This linkage works through the Health Information Exchange (HIE) to make PMP data available to providers via EHRs. The HIE facilitates electronic communication of patient information among organizations including hospitals and providers. In addition to the PMP, another resource to address the prescription drug abuse problem is the Emergency Department Information Exchange (EDIE), a web-based technology that specifically connects emergency departments statewide to track patients who visit multiple EDs. We also developed a connection between EDIE and PMP data through the HIE.

\section{Methods}

Increased provider utilization of the PMP will be achieved by using the HIE to create more seamless access to PMP data through providers' EHRs and through the EDIE system. This will be done by completing the build out of a transaction using NCPCP 10.6, piloting the connection with healthcare systems and EHR vendors, and by continuing to promote and encourage the PMP to remain an MU option through recent rule changes being proposed by CMS/ONC. The pilot with Epic was conducted in 2015 from April to October. Epic has released an update, available to Washington customers, that includes the connection between EHR and PMP. PMP data is also connected to EDIE. That connection is now live in 80 of 93 acute care hospital emergency departments.

\section{Results}

To date the transaction is in production with 80 emergency departments and achieving positive results. In 2015 the PMP received more than 2 million queries from the EDIE system via the HIE, compared to 900,000 queries via the online PMP portal in the year before the link through the HIE was available. We have also finished a pilot with a major EHR vendor and are working to on-board their customers. We are also working directly with healthcare systems, and as of September 2016 there are 3 healthcare facilities in testing that are expected to go live by the end of the year. Over 90 registrations for meaningful use of the PMP have been received, representing more than 1000 clinics. Improved access to PMP data benefits providers by allowing them to check the history of transactions linked to their DEA numbers, which can alert them to fraudulent prescriptions.

\section{Conclusions}

Integration of PMP data with other information systems will greatly enhance the accessibility and impact of the data. Making a connection to EDIE alone more than doubled the number of queries we received from providers in 2015. We anticipate even more inquiries once additional care settings are connected. We hope from this to see a continued decline in unintentional poisonings due to prescription drugs.

\section{Keywords}

HIE; Monitoring; overdose

\section{Acknowledgments}

This work was funded in part by an appointment to the Applied Public Health Informatics Fellowship Program administered by CSTE and funded by the Centers for Disease Control and Prevention (CDC) Cooperative Agreement 3U38-OT000143-01S4, by the Office of the National Coordinator for Health Information Technology Standards \& Interoperability Framework, and by federal financial participation from the Center for Medicare and Medicaid Services.

\section{*Dan Bolton}

E-mail: dan.bolton@doh.wa.gov 